Classification

Physics Abstracts

61.43.-j-61.16.-d

\title{
Order and Disorder in Boron Phases
}

\author{
Paola Favia $\left({ }^{1}\right)$, Tiziana Stoto $\left({ }^{1, *}\right)$, Michel Carrard $\left({ }^{1}\right)$, Pierre-André Stadelmann $\left({ }^{2}\right)$ \\ and Libero Zuppiroli $\left({ }^{1}\right)$ \\ ( $\left.{ }^{1}\right)$ Laboratoire de Physique des Solides Semi-Cristallins, École Polytechnique Fédérale, \\ 1015 Lausanne, Switzerland \\ $\left({ }^{2}\right)$ Centre Interdépartemental de Microscopie Électronique, École Polytechnique Fédérale, \\ 1015 Lausanne, Switzerland
}

(Received October 4; accepted December 3, 1996)

\begin{abstract}
The complicated rhombohedral unit cell of crystalline $\beta$-boron containing 105 atoms is a good approximant structure for a quasicrystal. Thus one may wonder if between the amorphous and the crystalline phases of boron, a non-metallic quasi-crystalline phase could exist. In order to examine this possibility and to characterize order and disorder in boron phases, we have performed high resolution electron microscopy (HREM) and electron energy loss spectroscopy (EELS) measurements. In the crystalline phase, the creation of large number of microtwins and stacking faults is the way that nature chooses to disorder boron. The presence of these microtwins seems to be the major obstacle towards the formation of a quasicrystal. A comparison between energy loss near edge structures and electronic density of states for different boron compounds has allowed us to identify the signature of the icosahedra in EELS measurements. In the amorphous phase, a special processing of HREM images proposed by Fan and Cowley has been applied in order to estimate the extent of local order in amorphous boron. Both techniques (HREM and EELS) have confirmed the presence of icosahedral units in the amorphous phase. This raises the question of the existence of a quasi-crystalline phase.
\end{abstract}

\section{Introduction}

Boron is a material with remarkable features. It has four crystalline modifications. The most stable one, the $\beta$-rhombohedral boron, has a unit cell of 105 atoms [1]. This material can also be obtained in an amorphous state by chemical vapour deposition or evaporation. All the crystalline boron polymorphs have the same structural unit: a boron icosahedron, $\mathrm{B}_{12}$. Diffraction studies have shown the presence of this unit in the amorphous phase, as well [2-5]. This natural tendency to form local fivefold symmetry is due to a particular covalent structure of boron, called threecentre bond, in which the electronic density is maximum in the centre of the boron triangle. This distinctive bonding gives the material exceptional thermal, electronic and transport properties [6].

$\left(^{*}\right)$ Present address: Industrial Research Ltd., Superconductivity, Electron Microscopy, Gracefield, PO box 31-310, Lower Hutt, New Zealand 
$\beta$-rhombohedral boron has a rhombohedral unit cell with $a=10.139 \AA$ and $\alpha=65.20^{\circ}$ [7]. This structure can be described as two sets of $\mathrm{B}_{12}$ icosahedra units, one set centred at the vertices of the rhombohedral unit and the other in the middle of neighbouring vertices [8]. Along the diagonal of the rhombohedral unit cell, one finds a $B_{28}-B-B_{28}$ chain. The $B_{28}$ units can be derived from the partial condensation of three $B_{12}$ icosahedra. Consequently, $B_{12}$ icosahedron can be regarded as the basic structural unit of the $\beta$-rhombohedral boron. It should be emphasised that none of the icosahedral units described above are perfectly regular, but slightly distorted.

Molecular orbital considerations of the boron $\mathrm{B}_{12}$ unit have shown that, among the 36 valence electrons of a boron icosahedron, 12 are pointing towards external bonds along the fivefold axis of this polyhedron [9]. Therefore, in a curved 3D space, boron would realize the paving of the space by icosahedra [10]. But in our Euclidean real space, $\beta$-boron crystallises in the trigonal system, which represents the best structural compromise for filling the space with icosahedral blocks, with 6 external bonds along the fivefold axis.

These considerations show that $\beta$-boron and related compounds appear to be good candidates for the first non-metallic quasicrystal, with hopefully new physical properties in a quasi periodic matrix. Indeed, recent studies have shown that it is theoretically possible to construct a model of icosahedral boron phase by decorating a 3D Penrose tiling of two rhombohedra, one being close to the unit cell of the $\beta$-boron crystal $[11,12]$. Experimentally, Kimura and his co-workers have made several attempts to obtain a quasicrystalline phase of boron by evaporating and subsequently heating it, but without any success $[13,14]$.

Schmirgeld et al. [15] have tried to disorder $\beta$-boron crystals by ion irradiation in order to obtain new structural forms with fivefold symmetry. However, even with very high doses by which every atom was displaced more than 35 times, no amorphization was observed. More striking was the lack of condensation of point defects. A recent study has shown that this remarkable self-healing has its origin in the unusual electronic structure and structural stability of the boron icosahedron [16].

It appears therefore that boron is a strange material: it exists in four crystalline modifications and an amorphous one, all having the same icosahedral structural unit. However, the transition from the crystalline to amorphous phase seems to be impossible. Therefore the following question is still open: is it possible in boron to obtain new intermediate phases between the crystalline and the amorphous states and, in particular, to obtain a new quasicrystalline phase? The aim of this study is to answer this question by studying the local order ranging from the crystal to the amorphous phase.

In the crystalline form, we have studied the numerous twin defects and their relations to the local fivefold symmetry. We have shown that these defects allow the trigonal symmetry to completely dominate. We have also studied the structure of some nanocrystals found in the amorphous sample which presumably appear in the first stage of crystallisation. We have observed that the complex structure of $\beta$-boron is already present in these nanocrystals.

In the amorphous state, we have studied the local order by high resolution electron microscopy (HREM) and electron energy loss spectroscopy (EELS). Although several works have already been done in this field, we have tried to go as far as possible in each technique. In particular, we have applied a numerical method to analyse high resolution images developed by Fan and Cowley [17] which, to our knowledge, has never been applied, except to the classical case of carbon. This technique allowed us to retrieve the icosahedral local arrangement in the amorphous phase and to give an evaluation of the extent of the local order. We have shown also, by comparing energy loss spectra and density of states of several borides, that a signature of the icosahedra can be found in the energy loss near edge structures (ELNES). 


\section{Experimental}

Thin foils of sintered $\beta$ boron and boron carbides were prepared from diamond-cut slices, $0.2 \mathrm{~mm}$ thick, with mechanical polishing, dimple grinding and argon ion milling. Commercial powder of amorphous boron (Armar product, $99.999 \%$ of purity) and powders of $\mathrm{LaB}_{6}$ and $\mathrm{CaB}_{6}$ were deposited on carbon coated grids after crushing in a boron carbide mortar.

High resolution electron microscopy was carried out at $300 \mathrm{keV}$, using a Philips EM430ST microscope with a point to point resolution of $0.2 \mathrm{~nm}$ and a spherical aberration constant of $1.2 \mathrm{~mm}$. Calculations of HREM images were carried out by using the software package EMS [18]. An absorption coefficient of 0.1 was included and a Debye-Waller temperature coefficient of $0.005 \mathrm{~nm}^{2}$ was used. The temporal and the spatial coherence were evaluated experimentally: an energy spread of $3 \mathrm{eV}$, corresponding to a defocus spread of $16 \mathrm{~nm}$, was measured using a Gatan 607 EELS spectrometer, while the beam convergence angle was of $1.6 \mathrm{mrad}$. The objective aperture diameter was $20 \mathrm{~mm}^{-1}$.

Electron energy loss spectroscopy was carried out at $200 \mathrm{keV}$, using a Hitachi HF- 2000 electron microscope equipped with a cold field emission gun and connected to a parallel analyser (Gatan 666 PEELS) interfaced to a Macintosh IIfx computer used as a multichannel analyser. Spectra were acquired with a dispersion of about $0.3 \mathrm{eV}$ per channel (resolution of $0.45 \mathrm{eV}$ ) using a collection angle of $50 \mathrm{mrad}$, a convergence angle of $8 \mathrm{mrad}$ and a spectrometer aperture of $3 \mathrm{~mm}$. We have verified that this large collection angle has no influence on the EELS features due to non-dipole effects. Spectra acquisition and treatment were performed using Gatan EL/P software (version 2.0). Spectra were taken from several large regions of all the samples in order to avoid artefacts due to structural anisotropy. The specimen thicknesses were always less than half of the mean free path in order to avoid plurial scatterings. Plasmon regions have not been studied.

\section{Results and Discussion}

Figure 1 shows the typical aspect of $\beta$-boron observed by conventional and high resolution electron microscopy. A high density of planar defects is evident. Analysis of diffraction patterns and simulations of high resolution images show that these defects are twin boundaries and stacking faults corresponding to the $\{100\}$ planes, i.e. the faces of the rhombohedral unit cell. The bright dots in Figures $1 \mathrm{~b}$ and $1 \mathrm{c}$ correspond to the icosahedra located at the vertices of the rhombohedral unit cell. The simulations of high resolution images confirm that twin boundaries pass through the centre of the icosahedral units, in agreement with previous investigations [19].

Extensive twinning has also been observed in other icosahedral boron compounds like $\alpha$-rhombohedral or boron carbides (see Hoard et al. [8]). This phenomenon is therefore closely related to the presence of the icosahedron. Indeed, the ease of formation of the twin boundaries can be explained by the fact that $\{100\}$ are mirror planes for the $B_{12}$ unit and the bondings between the $\mathrm{B}_{12}$ and $\mathrm{B}_{28}$ units are similar on each side of the twin boundaries. Therefore the deformation of the crystal due to the twin formation is minimal. Similar considerations apply also to the stacking faults that can be viewed as a pair of juxtaposed twin defects.

In some cases, the origin of these twin boundaries can be attributed to the plastic deformation occurring during the preparation of the samples by hot pressing. But, whatever their mechanism of formation, twin boundaries contribute notably to the structural disorder of the boron crystal and, consequently, can affect its physical properties. In particular, the ease of their formation allows the crystal to accommodate any plastic deformation and internal strains and to preserve the trigonal symmetry, preventing the formation of other phases.

HREM observations of the so-called amorphous boron material show the presence of some nanocrystals with typical sizes of $10 \mathrm{~nm}$ (Fig. 2). Interplanar distances and angles between planes 

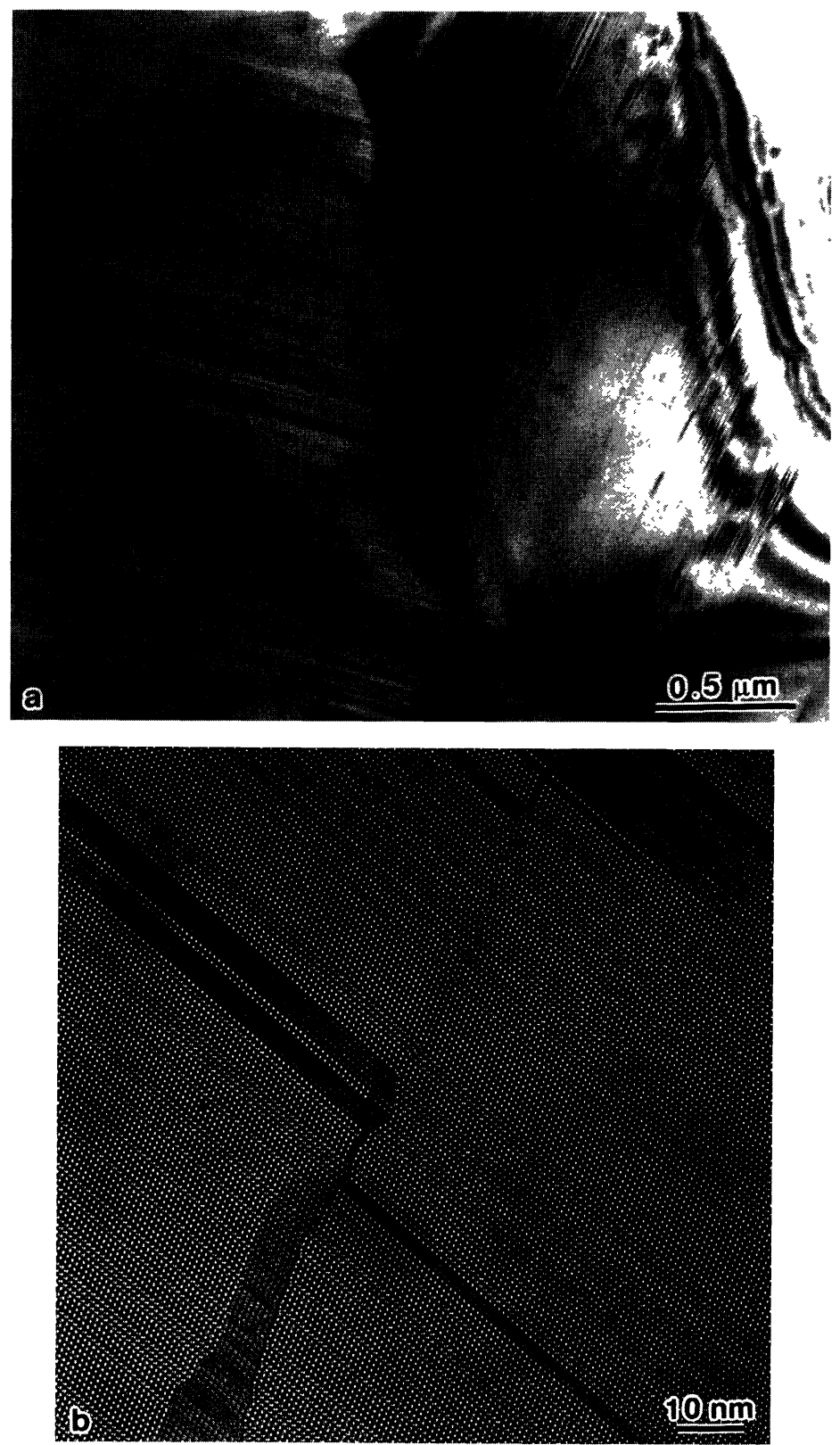

Fig. 1. - a) Low magnification bright field image of $\beta$-boron exhibiting a high density of twin boundaries and stacking faults. b) and c) High resolution transmission micrograph of $\beta$-boron. Twins and stacking faults are visible along the rhombohedral faces.

can always be interpreted with the structure of the $\beta$-boron and there is no evidence of a quasicrystalline order. Although the origin of these nanocrystals is uncertain, their existence suggests that trigonal crystalline order prevails on five-fold symmetry in the icosahedra arrangements. Furthermore, it suggests that the medium range order in the amorphous state is closed to the structure 


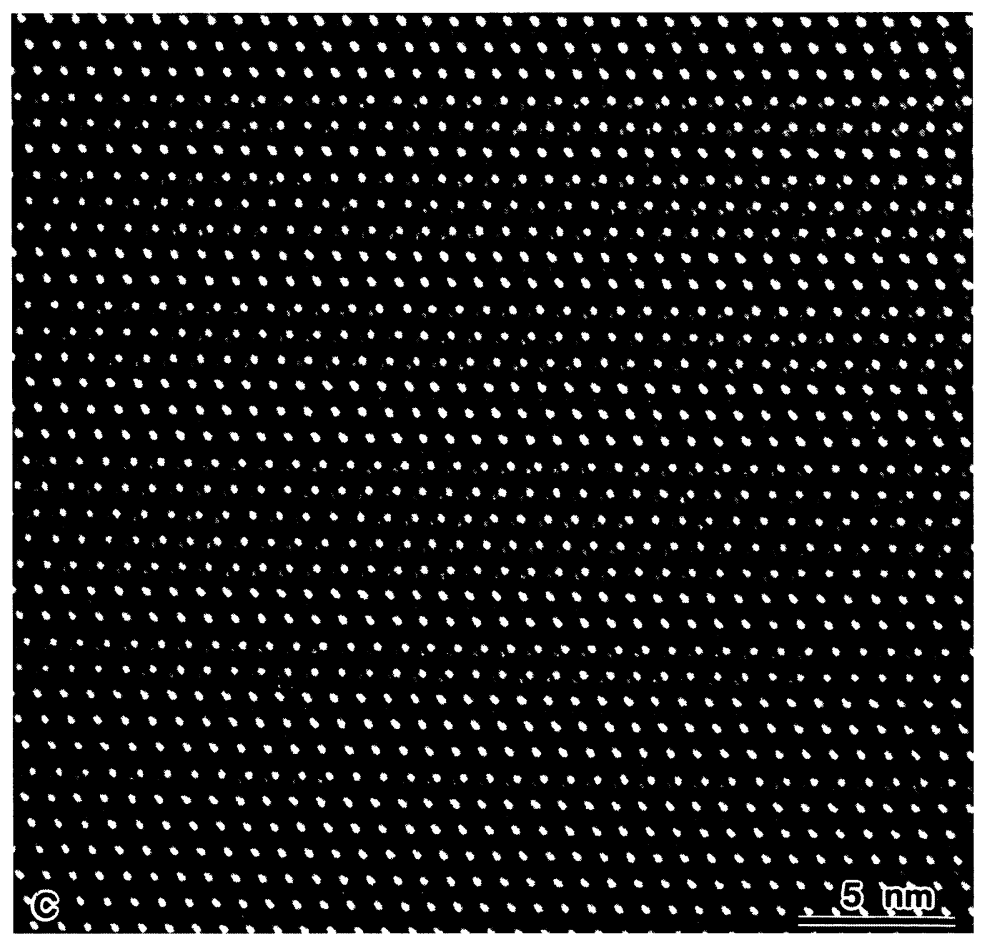

Fig. 1. - (continued.)

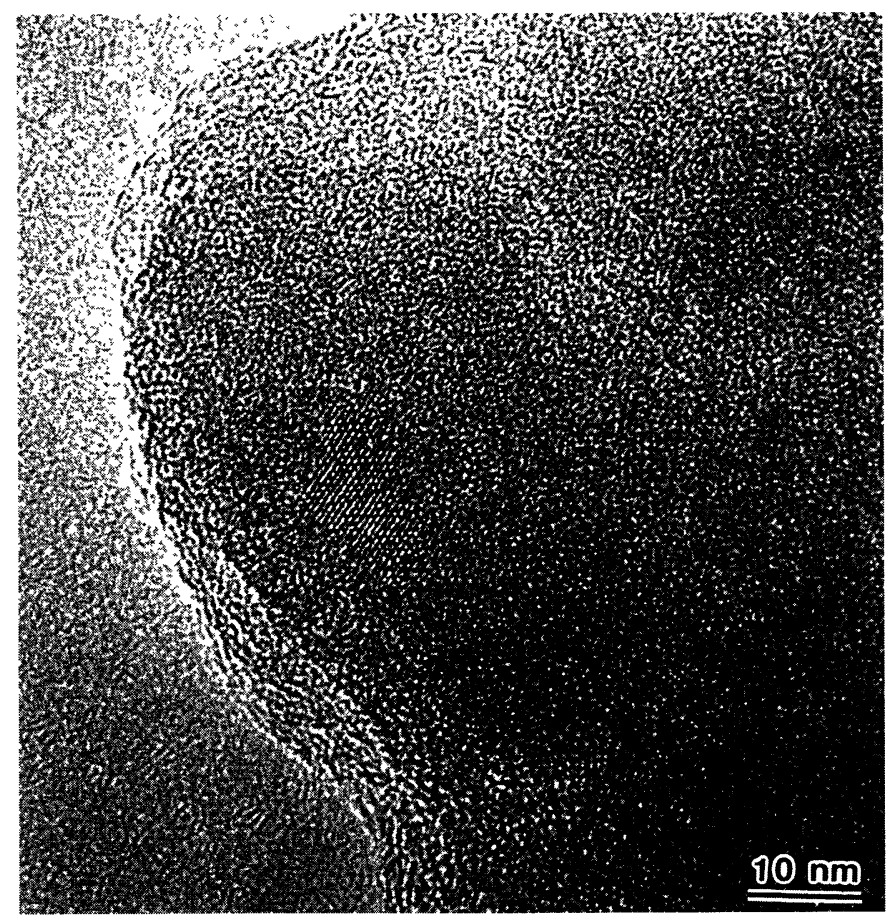

Fig. 2. - High resolution electron micrograph of a nanocrystal embedded in amorphous boron. 

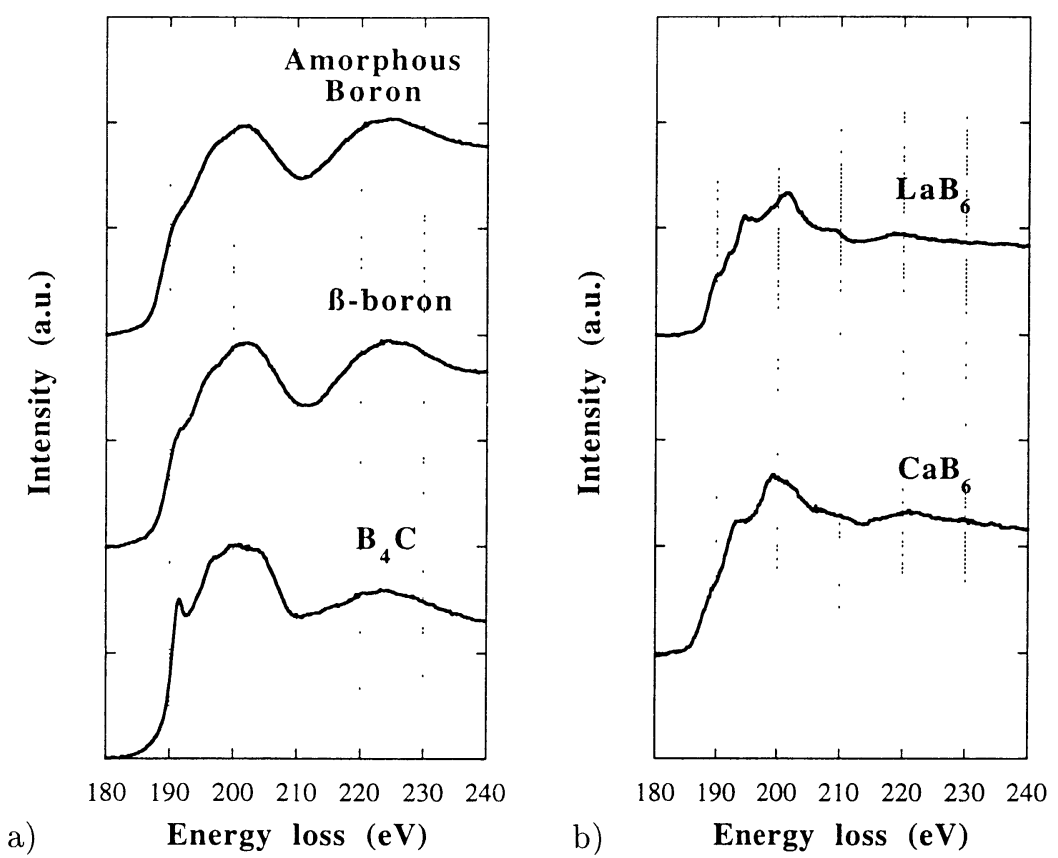

Fig. 3. - Electron energy loss spectra in the boron K-shell region of (a) amorphous boron and icosahedral borides $\beta$-boron and $\mathrm{B}_{4} \mathrm{C}$, and (b) non-icosahedral borides $\mathrm{LaB}_{6}$ and $\mathrm{CaB}_{6}$.

of $\beta$-boron. This corroborates with the observation of some $\beta$-boron crystallites in amorphous boron prepared by rapid quenching [20].

The study of energy loss near-edge structures (ELNES) of boron inner shell excitations in amorphous boron and different borides in the crystalline state has been carried out in order to obtain information of the local order in the amorphous state. All the details about this technique are very well described in the literature [21]. The identical ELNES features in different compounds allow the rapid determination of the local atomic environment even in complex structures. For example, Sauer et al. [22] have applied it to minerals in order to differentiate between planar-trigonal and tetrahedral coordinations of boron with oxygen.

The energy loss spectra in the boron K-shell region of amorphous boron are compared in Figure $3 \mathrm{a}$ with $\beta$-boron and $\mathrm{B}_{4} \mathrm{C}$ crystals, which have both $\mathrm{B}_{12}$ icosahedra as a structural unit. These spectra are almost identical. This is not expected since amorphous and crystalline carbon show different K-edge features [23]. Figure $3 \mathrm{~b}$ shows K-shell regions of borides which have a different structural unit: $\mathrm{LaB}_{6}$ and $\mathrm{CaB}_{6}$ with an octahedral unit. The near edge structures are drastically different from those of icosahedral borides. This shows that short range order of the icosahedral type exists in the amorphous compound. Similar results have been obtained by Suzuki, Tomita and Hayashi [24] on amorphous boron filaments grown by electron beam irradiation. Although the icosahedral borides show similar energy loss spectra, the sharpness of the peak observed at $192 \mathrm{eV}$ differs. This difference for $\mathrm{B}_{4} \mathrm{C}$ is probably due to the carbon atoms located either in the linear chain filling the rhombohedral unit cell or in the icosahedral unit.

The results of the ELNES studies can be interpreted in terms of density of states. Indeed, in this technique, core electrons are ejected to empty states just above the Fermi level. Therefore the near edge K-edge intensity is proportional to the projected p-like density of the final states [21]. Bullett $[25,26]$ has calculated the density of states below the Fermi level for many types of borides. 
a)

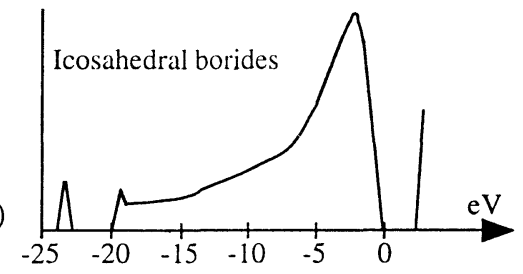

b)

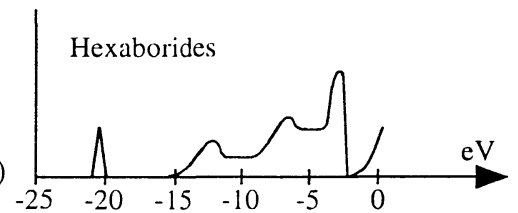

Fig. 4. - Schematic representation of the density of states (from Bullett $[25,26]$ ) of (a) icosahedral borides, and (b) hexaborides.

The different modifications of pure boron ( $\alpha$-rh, $\beta$-rh and $\beta$-tet), which all have the icosahedron as structural unit, show the same general shape of the density of states schematically represented in Figure 4a. In the same way, other icosahedral borides which contain foreign atoms, such as $\mathrm{B}_{13} \mathrm{C}_{2}, \mathrm{~B}_{12} \mathrm{P}_{2}, \mathrm{AlB}_{12}$ and $\mathrm{YB}_{66}$, present the same parabolic shape of the density of states. It should emphasised that all these compounds have different unit cells and their only common point is the presence of the $\mathrm{B}_{12}$ icosahedron. On the contrary, the density of states of borides which have different structural units, like the hexaborides $\mathrm{LaB}_{6}$ and $\mathrm{CaB}_{6}$, show a quite different shape, as sketched in Figure 4b.

These differences and similarities between densities of states should certainly extend just above the Fermi level and therefore explain quite well the results of the ELNES studies. One can conclude therefore that the icosahedron gives a characteristic ELNES fingerprint that can be used to investigate the local order in different borides. In particular, this study confirms the presence of icosahedral arrangements in the amorphous state. However the extent of this local order still remains unknown.

In order to obtain direct information about the extent of local ordering in amorphous boron, HREM images have been analysed using a method proposed by Fan and Cowley [17]. It is known that in HREM images of amorphous materials, even for very thin films, local ordering in three dimensions can be disguised by the projection process. Nevertheless, information on the local order can be achieved by studying the autocorrelation function calculated on the two-dimensional intensity distribution of an electron micrograph. Under certain limiting conditions (very thin object, defocus close to Scherzer), this function is equivalent to a two-dimensional form of the Patterson function, i.e. a projected map of the inter-atomic vectors. When properly normalized, this function gives the probability of finding an atom relative to any given atomic site.

In general, the autocorrelation function calculated over a sufficiently large area $\left(\approx 100 \mathrm{~nm}^{2}\right)$ is radially symmetric indicating that any interatomic vector occurs with equal probability in all orientations. When taking into account smaller regions $\left(\approx 10 \mathrm{~nm}^{2}\right)$, the autocorrelation function presents fringes indicating local correlations. However, the fringes are differently oriented with respect to each other and the sum of all of them gives no correlation at all.

Fan and Cowley proposed to overcome this difficulty by dividing a micrograph in small squared regions and obtaining a local autocorrelation function for each of these small regions. These local autocorrelation functions are then rotated with respect to a reference one, until a maximum value of the cross correlation function is obtained. Summing the rotated autocorrelation functions, 

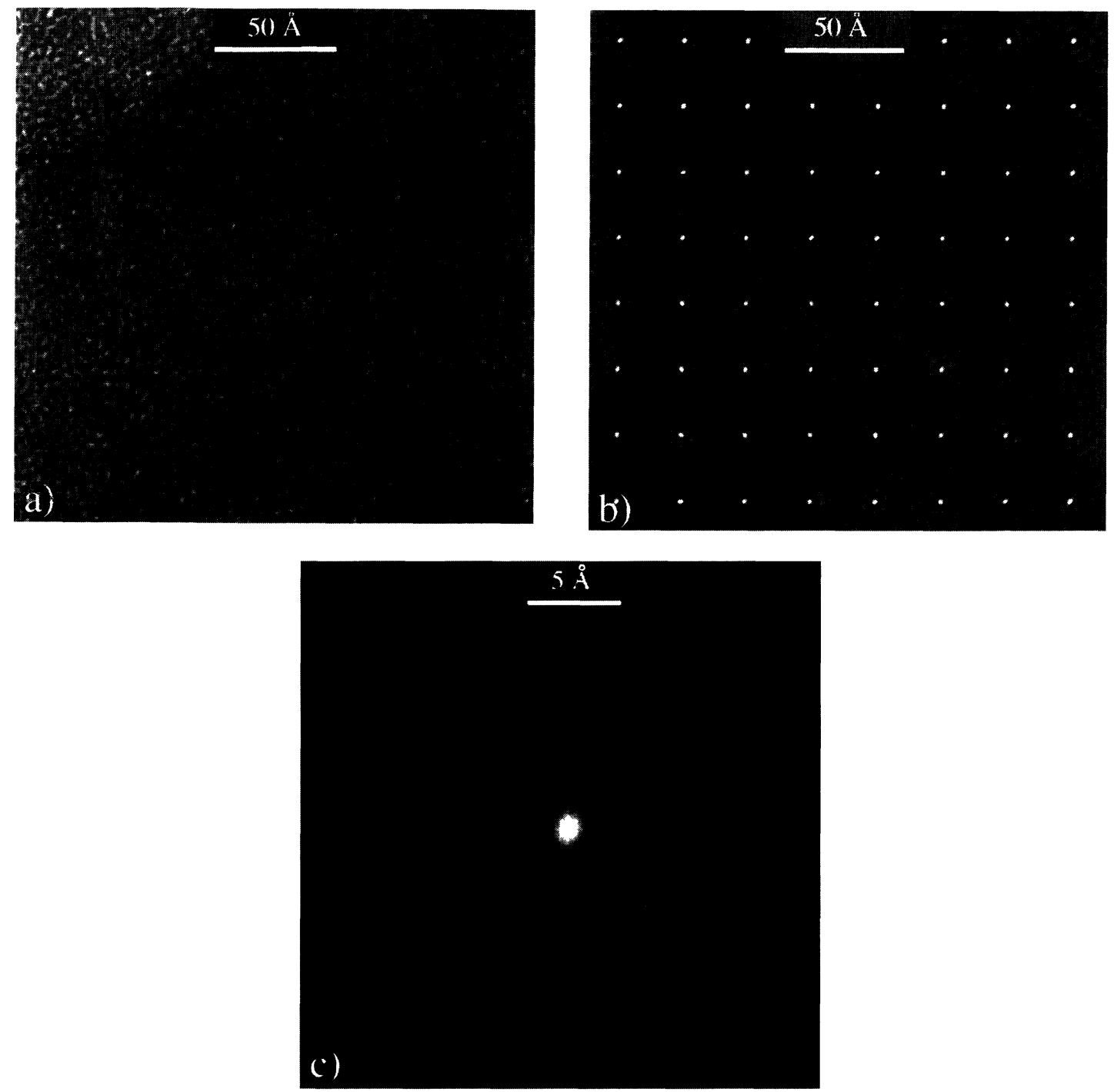

Fig. 5. - Processing of a high resolution image of amorphous boron following the method of Fan and Cowley [17]. a) Original micrograph, b) local autocorrelation functions, c) average local correlation function. The fringes give information on the projected inter-atomic distances. See text for details.

an average local correlation function is obtained, which provides a measure of the nature of the local order in the specimen. The size of the widest region showing the presence of fringes in the correlation function will give a measure of the extent of the local order.

In our investigation we have carried out the image processing for three different squared region sizes $\left(1.44 \times 1.44,2.8 \times 2.8\right.$ and $\left.5.7 \times 5.7 \mathrm{~nm}^{2}\right)$ as shown in Figure 5 for a $2.8 \times 2.8 \mathrm{~nm}^{2}$ region. A structure is evident in the calculated averaged local correlation function only for the 1.44 and $2.8 \mathrm{~nm}$ sizes, but it disappears for the $5.7 \mathrm{~nm}$ one. This indicates that the order extends over about $3 \mathrm{~nm}(\approx$ three unit cell of the $\beta$-boron crystal). The fringe pattern present in the averaged local autocorrelation function is shown in Figure $5 \mathrm{c}$. We have routinely employed the Fan-Cowley 


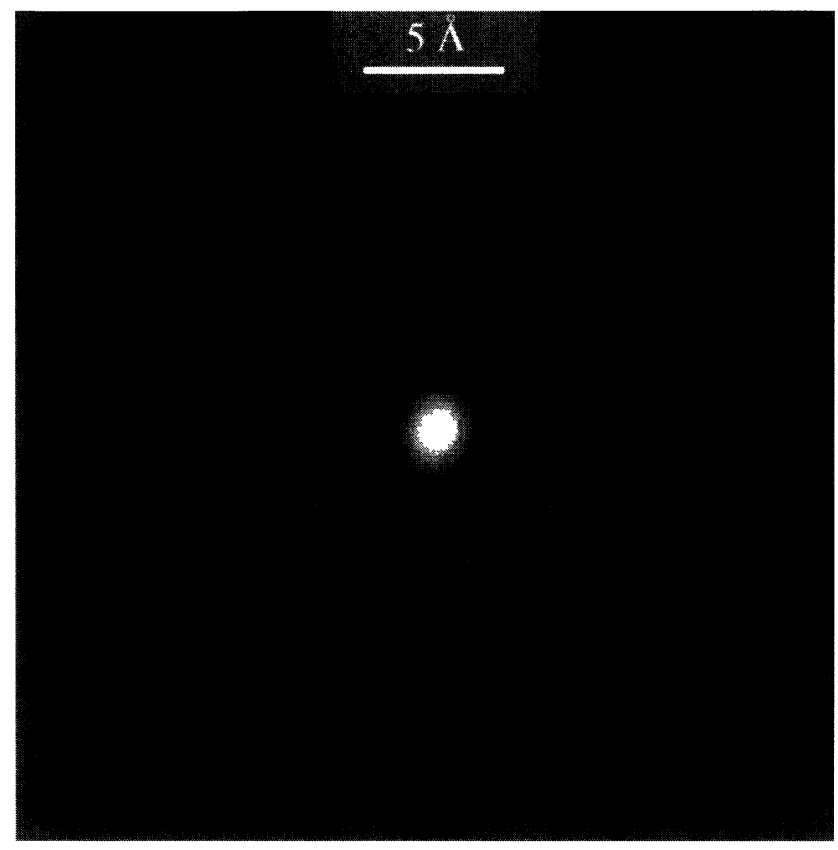

Fig. 6. - Comparison between the average local autocorrelation function of Figure $5 \mathrm{c}$ and a sketch of the two-dimensional Patterson function of the $\beta$-boron structure in the $\{001\}$ plane (each circle represents an icosahedron unit).

procedure, changing either the reference region in the same image, or the image itself in order to have a large set of data and hence a good statistics. As a result, we have found that distances between fringes range between 3.8 and 5.1 $\AA$. Even if these values represent only a projection of the interatomic distances, an important information comes from the upper limit $5.1 \AA$, which corresponds to the distance between two adjacent icosahedra in the crystalline boron. This confirms once again that amorphous boron is likely to contain $\mathrm{B}_{12}$ units.

Surprisingly, in Figure 5c, some spots can be seen suggesting some ordered structure. Figure 6 presents the same image with a sketch of the two-dimensional Patterson function of the $\beta$-boron structure in a $\{001\}$ plane superposed to it. Each circle represents an icosahedron unit. The similarity between both figures is striking. Even if such comparison is crude, this suggests that the local order in the amorphous state is close to that of $\beta$-boron in agreement with the results of Kobayashi [4].

\section{Conclusions}

Careful analysis of HREM images and ELNES spectra have allowed us to confirm the existence of the icosahedral unit in the amorphous state of boron. This unit is already present in all boron polymorphs. The omnipresence of this fivefold local order makes one believe that a quasicrystalline phase can be expected in this system.

However, the particular nature of the icosahedral unit seems to prevent this quasicrystalline phase. Its extreme resistance to irradiation precludes the use of this technique to disorder and reorder the crystalline phase. Its symmetry and bond-structure allow extensive twinnings of the crystal and enables it to accommodate any plastic deformation. 
Finally, the existence of some nanocrystals of $\beta$-boron embedded in amorphous boron, as well as some evidence of short range order close to the $\beta$-boron in the amorphous state, suggests that the trigonal symmetry of $\beta$-boron is energetically favoured.

\section{Acknowledgements}

We are grateful to Professors D. Emin and B. Morosin for giving us powders of borides for the EELS analysis. The present work was supported by the Swiss National Science Foundation under contract No. 20-39411.93.

\section{References}

[1] Bullett D.W., J. Phys. C 15 (1982) 415-426.

[2] Katada K., Jpn J. Appl. Phys. 5 (1966) 582-587.

[3] Badzian A. R., Mater. Res. Bull. 2 (1967) 987-992.

[4] Kobayashi M., J. Mater. Sci. 23 (1988) 4392-4398.

[5] Delaplane R. G., Dahlborg U., Howells W.S. and Lundström T., J. Non-Cryst. Solids 106 (1988) 66-69.

[6] Emin D., Phys. Today 40 (1987) 55-62.

[7] Callmer B., Acta Cryst. B 33 (1977) 1951-1954.

[8] Hoard J.L., Sullenger D. B., Kennard C. H. L. and Hughes R.E., J. Solid State Chem. 1 (1970) 268-277.

[9] Longuet-Higgins H.C. and Roberts M. de V., Proc. Roy. Soc. 230 (1955) 110119.

[10] Sadoc J.F. and Mosseri R., J. Phys. France 25 (1984) 1025.

[11] Weygand D. and Verger-Gaugry J.-L., Proc of the 13th Int. Congress on Electron Microscopy, B. Jouffrey and C. Colliex, Eds. (Les Éditions de Physique, les Ulis, 1994) pp. 761-762.

[12] Weygand D. and Verger-Gaugry J.L., C.R. Acad. Sci. Paris Série IIb 320 (1995) 253-257.

[13] Takeda M., Kimura K., Hori A., Yamashita H. and Ino H., Phys. Rev. B 48 (1993) 13159-13161.

[14] Kimura K., Hori A., Takeda M., Yamashita H. and Ino H., J. Non-Cryst. Solids 153\&154 (1993) 398-402.

[15] Schmirgeld L., Zuppiroli L., Brunel M., Delafon J. and Templier C., Proc. of the 10th Int. Symp. on Boron, Borides and related compounds., D. Emin, T. Aselage, A.C. Switendick B. Morosin and C.L. Beckel, Eds. (AIP Conf. Proc 231, New York, 1991) pp. 630-638.

[16] Carrard M., Emin D. and Zuppiroli L., Phys. Rev. B 51 (1955) 11270-11274.

[17] Fan G. Y. and Cowley J. M., Ultramicroscopy 17 (1985) 345-356.

[18] Stadelmann P. A., Ultramicroscopy 21 (1987) 131-146.

[19] McKelvy M. J., Smith A. R. R. and Eyring L., J. Solid State Chem. 44 (1982) 374-381.

[20] Galasso F., Vaslet R. and Pinto J., Appl. Phys. Lett. 8 (1966) 331-332.

[21] Egerton R.F., Electron Energy Loss Spectroscopy in the Electron Microscope (New York: Plenum, 1986).

[22] Sauer H., Brydson R., Rowley P.N., Engel W. and Thomas J.M., Ultramicroscopy 49 (1993) 198-209.

[23] Berger S.D., McKenzie D.R. and Martin P.J., Philos. Mag. Lett. 57 (1988) 285-290.

[24] Suzuki S., Tomita M. and Hayashi T., Jpn J. Appl. Phys. 34 (1995) L191-L194.

[25] Bullett D.W., Proc. of the Int. Conf. on the Physics and Chemistry of Boron and Boron-Rich Borides, D. Emin, T. Aselage, C.L Beckel, I.A. Howard and C. Wood, Eds. (AIP Conf. Proc. 140, New York, 1986) pp. 249-259.

[26] Bullett D.W., Proc. of the 11th Int. Symp. on Boron, Borides and related compounds, R. Uno and I. Higashi, Eds. (JJAP Series 10, Tokyo, 1994) pp. 31-34. 